CARTA AL EDITOR

\section{CONSIDERACIONES SOBRE LA EVALUACIÓN EN CONDICIONES DE CAMPO DE UNA PRUEBA SEROLÓGICA RÁPIDA PARA DETECTAR ANTICUERPOS IgM E IgG CONTRA SARS-COV-2}

\section{CONSIDERATIONS ON THE EVALUATION UN- DER FIELD CONDITIONS OF A RAPID TEST FOR DETECTION OF IGM AND IGG ANTIBODIES AGAINST SARS-COV-2}

\author{
Carmela Ganoza Arenas (ib) 1,a, Juan José Montenegro- \\ Idrogo (10) ${ }^{2, b}$, Alfredo Juan Chiappe-Gonzalez (10) ${ }^{2, b}$, \\ Juan Carlos Gómez de la Torre (i] ${ }^{3, c}$

\footnotetext{
Asociación Civil IMPACTA, Lima, Perú. Nacional Dos de Mayo, Lima, Perú. especialista en Infectología; ${ }^{c}$ médico especialista en Patología Clínica e Infectología.
} \\ 2 Servicio de Medicina de Enfermedades Infecciosas y Tropicales, Hospital \\ 3 Facultad de Medicina Humana, Universidad Ricardo Palma, Lima, Perú. \\ a Médico cirujano, especialista en Patología y Laboratorio Clínico; ${ }^{\text {b }}$ médico
}

Sr. Editor, al leer detenidamente el artículo de Vidal-Anzardo et al. tenemos algunas observaciones que plantear sobre el uso de una prueba rápida para detectar anticuerpos IgM/IgG contra SARS-CoV-2 en condiciones de campo ${ }^{(1)}$, donde la prueba COVID-19 IgG/IgM Rapid Test Cassette (whole blood/serum/ plasma. Zhejiang Orient Gene, Biotech Co., LTD o «prueba rápida»), utilizada como herramienta de apoyo diagnóstico en dicha publicación, tendría cierto potencial para definir la presencia de anticuerpos anti-SARS-CoV-2 en las muestras estudiadas.

En el artículo no se detalla la referencia de los estudios de validación o de control de calidad internacional y local de las pruebas utilizadas, idealmente estos deberían estar a cargo de entidades reguladoras del estado o terciarias para mayor objetividad y con ello disminuir el error de interpretación de los resultados ${ }^{(2)}$. Además, el primer artículo que evalúa la prueba de Zhejiang por terciarios se publicó el 14 de abril de $2020^{(3)}$, días antes de conocer la falta de licenciamiento en su país de origen ${ }^{(4)}$.

Citar como: Ganoza C, Montenegro-Idrogo J, Chiappe-Gonzalez A, Gómez J. Consideraciones sobre la evaluación en condiciones de campo de una prueba serológica rápida para detectar anticuerpos IgM e IgG contra SARS-CoV-2. Rev Peru Med Exp Salud Publica. 2020;37(3):571-2. doi: https://doi.org/10.17843/ rpmesp.2020.373.5753.

Correspondencia: Juan José Montenegro Idrogo; Av. Arnaldo Márquez 579, int 507, Jesús María, Lima, Perú; montenegroidrogo@gmail.com

Recibido: 07/05/2020 Aprobado: 13/05/2020 En línea: 17/08/2020
Sobre la interpretación metodológica en pruebas diagnósticas, la sensibilidad de una prueba debe probarse en personas con mayor certeza de infección y la especificidad en no infectados y en personas con patologías de espectro clínico similar (probabilidad preprueba). La prueba molecular de reacción en cadena de la polimerasa (PCR) para SARS-CoV-2 es con frecuencia utilizada como el estándar de oro para diagnosticar esta infección, pero tiene limitaciones que dependen del tiempo de enfermedad, origen de la muestra, entre otros ${ }^{(5,6)}$. Así, el diagnóstico estándar debe entenderse como la suma de criterios clínico-radiológico-epidemiológicos y pruebas etiológicas (PCR y/o serología). No obstante, el artículo de Vidal-Anzardo et al. ${ }^{(1)}$ describe la sensibilidad de la prueba rápida en pacientes con tres perfiles no homogéneos en cuanto a certeza de diagnóstico o descarte de infección (hospitalizados, trabajadores de salud y casos sospechosos), y establece la especificidad a partir de sueros de pacientes con enfermedades clínicamente heterogéneas, no necesariamente parecidas a la COVID-19 y sin inclusión de individuos sanos. A pesar de los ajustes de homogenización, la interpretación de la prueba se ve limitada por su temporalidad, que evidencia desigualdad del tamaño de muestra según grupos evaluados por esta variable ${ }^{(5,6)}$.

Los estudios citados en el artículo determinaron positividad de PCR al repetirlo en muestras negativas, mientras que en el estudio ${ }^{(1)}$ se consideró negatividad con un solo resultado, lo cual es una limitación importante, ya que el hisopado nasal depende de la técnica de la toma de muestra y el rendimiento ${ }^{(6)}$. Además, en los casos con ambas pruebas negativas no se excluyeron otras etiologías respiratorias estacionales. Los resultados con los que se compara el artículo distan mucho en cuanto al rendimiento y reportan amplios intervalos de confianza que podrían explicarse por la heterogeneidad de tamaños en los grupos de tiempo evaluados.

Por ello, consideramos que con los resultados mostrados no se puede concluir que existe un rendimiento diagnóstico adicional con relación a la prueba molecular, pero puede servir como instrumento complementario al apoyo diagnóstico, enfatizando que su negatividad no descarta la infección, por lo cual debe primar la suma de criterios, en especial el clínico-radiológico.

Contribuciones de autoría: CGA, JMI, ACG, JGT participaron en la concepción de la idea, redacción del artículo, lectura crítica y aprobación de la versión final.

Fuentes de financiamiento: Autofinanciado.

Conflictos de interés: Los autores declaran que no tienen conflicto de interés alguno. 


\section{REFERENCIAS BIBLIOGRÁFICAS}

1. Vidal-Anzardo M, Solis G, Solari L, Minaya G, Ayala-Quintanilla B, Astete-Cornejo J, et al. Evaluación en condiciones de campo de una prueba serológica rápida para detección de anticuerpos $\operatorname{IgM}$ e IgG contra SARS-COV-2. Rev Peru Med Exp Salud Pública. 2020;37(2):17. doi: $10.17843 /$ rpmesp.2020.372.5534.

2. Igloi Z, Hashem Abou-N ZAK, Okba N, Geurts van Kessel C, Koopmans M. Clinical sensitivity and specificity of three rapid SARSCoV-2 Antibody (IgM/IgG) Tests on a hospitalized patient cohort: InTec, Cellex and Orient Gene [Internet]. Países Bajos: Erasmus MC; 2020 [citado el 25 de abril de 2020]. Disponible: https://www.confirmbiosciences.com/covid-19-resources/case-study-product-comparison-who-erasmusmc-viroscience/.
3. Hoffman T, Nissen K, Krambrich J, Ronnberg B, Akaberi D, Esmaeilzadeh M, et al. Evaluation of a COVID-19 IgM and IgG rapid test; an efficient tool for assessment of past exposure to SARS-CoV-2. Infection Ecology \& Epidemiology. 2020;10(1). doi: $10.1080 / 20008686.2020 .1754538$.

4. Nbcnews. Unapproved Chinese coronavirus antibody tests being used in at least 2 state [Internet]. Nueva York: NBC News Digital; 1996 [citado el 25 de abril de 2020]. Disponible en: https://www.nbcnews. com/health/health-news/unapproved-chinese-coronavirus-antibody-tests-being-used-least-2-states-n1185131.

5. Lou B, Li T, Zheng S, Su Y, Li Z, Liu W, et al. Serology characteristics of SARS-CoV-2 infection since the exposure and post symptoms onset. medRxiv. 2020. doi: 10.1101/2020.03.23.20041707.

6. Wang W, Xu Y, Gao R, Lu R, Han K, Wu G, et al. Detection of SARSCoV-2 in Different Types of Clinical Specimens. JAMA. 2020. doi: 10.1001/jama.2020.3786. 